\title{
Thermodynamic Properties of the Harmonic Oscillator and a Four Level System
}

\author{
Oladunjoye A. Awoga \\ Theoretical Physics Group, Department of Physics, University of Uyo, Uyo, Nigeria \\ E-mail: ola.awoga@yahoo.com
}

Akpan N. Ikot

Theoretical Physics Group, Department of Physics, University of Uyo, Uyo, Nigeria

E-mail: ndemikot2005@yahoo.com

Aniesua A. Essiett

Theoretical Physics Group, Department of Physics, University of Uyo, Uyo, Nigeria

E-mail: aessiett2@yahoo.com

Louis E. Akpabio

Theoretical Physics Group, Department of Physics, University of Uyo, Uyo, Nigeria

E-mail: leabio2002@yahoo.com

Received: January 10, 2011 Accepted: January 21, 2011 doi:10.5539/apr.v3n1p47

\begin{abstract}
The thermodynamics properties of a quantum harmonic oscillator and four level oscillator systems are evaluated. These results lead to the exact value for the entropy of the system which corresponds to the second law of thermodynamics. We also show the numerical results for the harmonic and four level oscillators and it is in good agreement with the one obtained before in the literature.
\end{abstract}

Keywords: Density Matrix, Four Level System, Harmonic Oscillator, Heat Capacity, Entropy

\section{Introduction}

The formation of thermodynamics rests on the entropy, temperature and the three laws of thermodynamics, namely the first law, second law and third law, relating these state variables (Sethna 2006, Landau and Lifshitz 1980, Kittel 1988). However, the statistical mechanical foundation of thermodynamics relies strongly on the quantum mechanical properties of matter especially their characteristics at the low temperature regime.

However, the statistical mechanics gives rise to some subtlety when going from a closed description of all degrees of freedom, including those of large environments to a reduced description of an open system where all bath degrees of freedom are traced out. In recent times different definitions of specific heat have been discussed and proposed (Hanggi and Ingold 2006). And in addition the second law of thermodynamics in the quantum region by calculating the entropy $\mathrm{S}$ for a quantum oscillator in an arbitrary heat both at finite temperature have been examined (Hanggi and Ingold 2008, Ingold et al 2009, Hanggi and Ingold 2005).

The harmonic oscillator has played a significant role in physics and chemistry. The specific heat of crystals has been calculated for both Einstein and Debye approximation (Feynman 1972, Ingold et al 2009). The Debye theory predicts that the leading term in the heat capacity of all dielectric solids at sufficiently low temperature is to the order of $\mathrm{T}^{3}$. There are many physical properties that can be calculated in the harmonic models. Some of this includes thermal conductivity, thermal expansion obtained from the anharmonic terms in the crystal and many others (Kim et al 2003, Hijar and de Zarate 2010). 
The simplest way of analyzing the harmonic oscillator is to evaluate the partition function of the system. Many texts (Davydov 1991, Merzbacher 1976, Messiah 1970) have evaluated the partition function of harmonic systems because once the partition function of a system is known their thermodynamic properties can then be obtained.

In this paper, our objective is to evaluate the thermodynamics of the harmonic oscillator and a four level system by the method of density matrix and compare our results with those obtained via the partition function.

The organization of the paper is as follows; in section 2 we review the density matrix and section 3 we represent the dynamics of dissipative quantum system, in sections 4 we evaluate the thermodynamic properties of the harmonic oscillator. In section 5 we introduce the dynamics of a four level system; while in section 6 we calculate the thermodynamic properties of a four-level system while section 7 gives a brief discussion.

\section{Density Matrix}

In quantum mechanics, the state of an isolated system is represented by a state vector $\psi$ which can be expanded as

$$
\psi=\sum_{\alpha} a_{\alpha} \psi_{\alpha}
$$

where $a_{\alpha}=\left\langle\varphi_{\alpha} \mid \psi\right\rangle$ and $\left\langle\varphi_{\beta} \mid \varphi_{\alpha}\right\rangle=\delta_{\alpha \beta}$

The ensemble average of the expectation value of $\hat{A}$ is

$$
\begin{aligned}
\langle\hat{A}\rangle & =\sum_{i} P_{i} A_{i} \\
& =\sum_{i} P_{i}\left\langle\psi_{i}|\hat{A}| \psi_{i}\right\rangle
\end{aligned}
$$

where $p_{i}$ represent the probability.

The density operator is defined as

$$
\hat{\rho}=\sum_{i}\left|\psi_{i}\right\rangle P_{i}\left\langle\psi_{i}\right|
$$

and one obtain the ensemble average in terms of the density operator as (Hijar H, 2010)

$$
\begin{gathered}
\langle A\rangle=\sum_{i} p_{i}\left\langle\psi_{i}|\hat{A}| \psi_{i}\right\rangle \\
\langle A\rangle=\operatorname{Tr}(\hat{\rho} \hat{A})
\end{gathered}
$$

In order to normalize the density operator we write (4) in the form

$$
\langle A\rangle=\frac{\operatorname{Tr}(\hat{\rho} \hat{A})}{\operatorname{Tr}(\hat{\rho})}
$$

The canonical ensemble stipulates a density operator of the form

$$
\hat{\rho}=e^{-\beta \hat{H}}
$$

where $\beta=1 / K_{B} T$ and (5) becomes

$$
\langle A\rangle=\frac{\operatorname{Tr}(\hat{\rho} \hat{H})}{\operatorname{Tr}(\hat{\rho})}
$$




$$
\langle A\rangle=\frac{\sum_{n} e^{-\beta E_{n}} A_{i}}{\sum e^{-\beta E_{n}}}
$$

Where $Z=\sum_{n} e^{-\beta E n}$ and $P_{n}=\frac{e^{-\beta E_{n}}}{z}$

In terms of canonical density operator, we write

$$
\hat{P}=z^{-1} e^{-\beta H}, Z=\operatorname{Tr} e^{-\beta \hat{H}}
$$

The ensemble average for any observable is then given by (Landau and Lifshitz 1980 and Davydov 1991)

$$
\begin{aligned}
\langle f\rangle & =\operatorname{Tr}(\hat{\rho} f) \\
& =\frac{\operatorname{Tr} f e^{-\beta R}}{\operatorname{Tr} e^{-\beta R}}
\end{aligned}
$$

Having established a relation for the canonical density operator, we then obtain the internal energy, entropy and Helmholtz free energy in terms of this operator as

$$
\begin{gathered}
\langle E\rangle=\frac{\partial}{\partial \beta} \operatorname{In}\left[\operatorname{Tr}\left(e^{-\beta \hat{H}}\right)\right] \\
S=\left\langle-K_{B} \operatorname{In}[\hat{\rho}]\right\rangle \\
=-k_{\beta} \operatorname{TT}(\hat{\rho} \operatorname{In}[\hat{\rho}]) \\
F=\operatorname{Tr}(\hat{\rho} \hat{H})-\operatorname{TTr}(\hat{\rho} \hat{s})
\end{gathered}
$$

\section{Dynamics of Dissipative Quantum Systems}

The wave function $|\bar{\psi}\rangle$ is the state of a system in a pure quantum mechanics which is an element of a Hilbert space H. However, for a dissipative quantum system, a quantum statistical formulation is employed since dissipative effects can and do convert pure states into statistical ensembles. As was noted before, the state of the system is usually represented by a density operator $\hat{\rho}$ whose diagonal elements determine the population of the energy eigenstate while the off diagonal elements determine the coherence between energy eigenstates which distinguish coherent superposition states. In a non-dissipative system the time evolution of the density matrix $\hat{\rho}(t)$ with $\hat{\rho}\left(t_{0}\right)=\rho_{0}$ is governed by (Merzbacher 1970)

$$
\hat{\rho}(t)=\hat{u}(t) \rho\left(t_{0}\right) u^{+}(t)
$$

where $\hat{u}(t)$ is the time-evaluation operation satisfying our Schrödinger equation

$$
\begin{aligned}
i \hbar \frac{d}{d t} \hat{u}(t) & =\hat{H}(t) \hat{u}(t) \\
\hat{u}(0) & =\hat{\mathrm{I}}
\end{aligned}
$$

where $\hat{I}$ is the identity operator.

The density operator also satisfies the quantum Liouville equation

$$
i \hbar \frac{d}{d t} \hat{\rho}(t)=[\hat{H}, \hat{\rho}(t)]
$$

where $\mathrm{H}$ is the total Hamiltonian of the system which depends on a set of control field $f_{n}$ 


$$
\hat{H}=\hat{H}_{0}+\sum_{n=1}^{W} f_{n}(t) \hat{H}_{n}
$$

with $\hat{H}_{0}$ being the internal Hamiltonian and $\hat{H}_{n}$ is the interaction Hamiltonian for the field $f_{n}$ for $I \leq n \leq N$. Now substituting (15) into (14) we get

$$
i \hbar \frac{d}{d t} \hat{\rho}(t)=\left[\hat{H}_{0}, \rho(t)\right]+\sum_{n=1}^{N} f_{n}(t)\left[\hat{H}_{n} \hat{\rho}(t)\right]+i \hbar t_{0}[\hat{\rho}(t)]
$$

where $t_{0}$ is the dissipation super-operator.

\section{The Harmonic Oscillator}

Considering an oscillator with degree of freedom of unity, mass $m$ and frequency $\omega$, we write the Hamiltonian of the system as

$$
H=\frac{p^{2}}{2 m}+\frac{1}{2} m \omega^{2} x^{2}
$$

Using (7) we write the partition function as

$$
Z=\operatorname{Tr}\left(e^{-\beta \hat{H}}\right)
$$

yielding the well-known expression

$$
Z=\frac{1}{2} \operatorname{Cosec}\left(\frac{\beta \hbar \omega}{2}\right)
$$

with (9) we find the internal energy as

$$
\langle E\rangle=\frac{\hbar \omega_{0}}{2}+\frac{\hbar \omega_{0}}{\left(e^{\hbar \omega_{k T}}-1\right)}
$$

Figure (1) shows that there is a linear relationship between the average energy and temperature. This is in concordance with quantum mechanics with

$$
\langle n\rangle \approx \frac{\hbar \omega_{0}}{\left(e^{\hbar \omega}-1\right)}
$$

giving

$$
\langle E\rangle=\frac{\hbar w_{0}}{2}+\langle n\rangle
$$

where the leading term is the ground state energy.

The Helmhotz free energy is obtained using (11) as

$$
F=\frac{\hbar \omega}{2}+\frac{1}{\beta} \operatorname{In}\left[1-e^{-\beta \hbar \omega}\right]
$$

The free energy increases negatively with temperature which again agrees with the prediction of thermodynamics as shown in fig. (2)

Similarly, the entropy is calculated from (9)

$$
S=\frac{\hbar \omega}{T} \frac{e^{-\beta \hbar \omega}}{\left(1-e^{-\beta \hbar \omega}\right)}-k_{B} I n\left[1-e^{-\beta \hbar \omega}\right]
$$

For low temperatures in the region $\beta>>1$, the entropy approaches zero like (Landau and Lifshitz 1980) 


$$
S=\frac{\hbar \omega}{T} e^{-\beta \hbar \omega}
$$

The entropy approaches a constant value as temperature increases. This obeys the second law of thermodynamics which state that for a closed system $\Delta S \geq 0$ as shown in figure 3. The same as (E. Kozliak and F. L. Lambert, 2008).

We also calculate the specific heat capacity from (20) as

$$
\begin{gathered}
C v=\frac{\partial}{\partial T}\langle E\rangle \\
=k_{B}\left(\frac{\hbar \omega}{k_{B} T}\right)^{2} \frac{e^{-\beta \hbar \omega}}{\left(1-e^{-\beta \hbar \omega}\right)^{2}}
\end{gathered}
$$

At low temperatures $\beta>>\mathrm{I}$, the $\mathrm{C}_{\mathrm{v}}$ behaves as

$$
C_{v}=k_{B}\left(\frac{\hbar \omega}{K_{B} T}\right)^{2} e^{-\beta \hbar \omega}
$$

and this characteristic is not analytic in temperature and corresponds to Einstein's model for low-temperature behaviour of the $C_{V}$ of a solid. For high temperature $\hat{\beta}<<1$ we find (P. Hanggi, 2008)

$$
C_{v}=k_{B}\left[1-\frac{1}{12}\left(\frac{\hbar \omega}{k_{B} T}\right)^{2}+\varphi\left(T^{-4}\right)\right]
$$

and this shows that the limit of free particle is not obtained from the harmonic oscillator by setting $\omega \rightarrow 0$

The $\mathrm{C}_{\mathrm{v}}$ approaches a constant value i.e. $C_{v} \approx k_{B}$ as temperature increases. This is the Rule of Dulong and Petit in classical limit.

Equation (26) can also be written as an infinite series as

$$
C_{v}=k_{B}\left(\frac{\hbar \omega}{k_{B} T}\right)^{2} \sum_{n=1}^{\infty} n e^{-n \hbar \omega}
$$

The behaviour of each state is shown in figure 5 for equation (29).

\section{Dynamics of a Four-Level System}

The Hamiltonian for a driven for level system with energy level $t_{0}<t_{1}$ is (Merzbacher 1976)

$$
\hat{H}(t)=\hat{H}_{0}+f_{1}(t) \hat{H}_{1}+f_{2}\left(t_{0}\right) \hat{H}_{2}
$$

where $\hat{H}_{0}$ is the internal Hamiltonian of the system and $\hat{H}_{2}$ represent interaction Hamiltonian with independent real value control fields $f_{1}(t)$ and $f_{2}(t)$

$$
\begin{aligned}
& H_{1}=\alpha_{1}\left(\begin{array}{cccc}
0 & 0 & 0 & 1 \\
0 & 0 & 1 & 0 \\
0 & 1 & 0 & 0 \\
1 & 0 & 0 & 0
\end{array}\right) \\
& H_{2}=\alpha_{2}\left(\begin{array}{cccc}
0 & 0 & 0 & -i \\
0 & 0 & i & 0 \\
0 & -i & 0 & 0 \\
1 & 0 & 0 & 0
\end{array}\right)
\end{aligned}
$$

with $\alpha_{1}$ and $\alpha_{2}$ being dipole moments for the transition and the transition frequency

$$
\omega=\left(t_{1}-t_{0}\right) / \hbar
$$


Using (12), (14), (16) and (30) we write our Liouville equation in the form

$$
\begin{gathered}
\frac{d}{d t}|\rho(t)\rangle=t|\rho(t)\rangle \\
\left.=\left|(t h)^{-1}\left(t_{0}+f(t) t_{1}+f(t) t_{2}\right)+t_{0}\right| \rho(t)\right\rangle
\end{gathered}
$$

where

$$
\begin{aligned}
& |\rho(t)\rangle=\left(\rho_{11}(t), \rho_{12}(t) \cdots \rho_{n m}(t)\right)^{T} \\
& H_{0}=\left(\begin{array}{cccc}
0 & 0 & 0 & 1 \\
0 & -\hbar \omega & 0 & 0 \\
0 & 0 & \hbar \omega & 0 \\
0 & 0 & 0 & 0
\end{array}\right) \\
& t_{1}=\alpha_{1}\left(\begin{array}{cccc}
0 & -1 & 1 & 0 \\
-1 & 0 & 0 & 1 \\
0 & 0 & 0 & -1 \\
1 & 1 & -1 & 0
\end{array}\right) \\
& t_{2}=a_{2}\left(\begin{array}{cccc}
0 & -t & -t & 1 \\
t & 0 & 1 & -t \\
0 & 0 & 0 & -t \\
1 & t & t & 0
\end{array}\right) \\
& t_{0}=\left(\begin{array}{cccc}
-r_{21} & 0 & 0 & r_{12} \\
0 & -\Gamma & 1 & 0 \\
0 & 0 & -\Gamma & 0 \\
r_{21} & 0 & 0 & -r_{21}
\end{array}\right)
\end{aligned}
$$

with $r_{21}$ defining the rate of population relaxation from $|2\rangle$ to $|1\rangle$ while $r_{21}$ is the rate of population from $|1\rangle$ to $|2\rangle$ and $\Gamma$ defines the dephasing rate.

\section{The Thermodynamics of a Four-Level State System}

We now consider a system consisting of $\mathrm{N}$ independent components with four internal states described by a simple particle Hamiltonian of the form

$$
\hat{H}=\left(\begin{array}{cccc}
\epsilon_{0}-\epsilon_{1} & 0 & 0 & 0 \\
0 & \epsilon_{0}+\epsilon_{1} & 0 & 0 \\
0 & 0 & \epsilon_{0}+\epsilon_{1} & 0 \\
0 & 0 & 0 & \epsilon_{0}-\epsilon_{1}
\end{array}\right)
$$

where the system is in thermal equilibrium with a reservoir at temperature T. We construct the density matrix of the system as

$$
\rho=e^{-\beta H}
$$




$$
=\left(\begin{array}{l}
e^{\left.-9 ! \varepsilon_{2}-\varepsilon_{1}\right]} \\
0 \\
0 \\
0
\end{array}\right.
$$
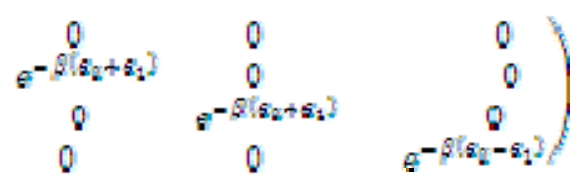

In order to normalize (37) we take its trace as

$$
\operatorname{Tr}(\rho)=1
$$

which yields

$$
C\left[e^{-\beta\left(\epsilon_{0}-\epsilon_{1}\right)}+e^{-\beta\left(\epsilon_{0}+\epsilon_{1}\right)}+e^{-\beta\left(\epsilon_{0}+\epsilon_{1}\right)}+e^{-\beta\left(\epsilon_{0}-\epsilon_{1}\right)}\right]=1 .
$$

Simplifying (38) gives the normalization constant $\mathrm{C}$ as

$$
C=\frac{e^{\beta \epsilon_{0}}}{4 \cosh \left(\beta \epsilon_{1}\right)}
$$

and the density matrix (37) becomes

$$
\rho=\frac{1}{4 \cosh \left(\beta \epsilon_{1}\right)}\left(\begin{array}{cccc}
e^{\beta \epsilon_{1}} & 0 & 0 & 0 \\
0 & e^{-\beta \epsilon_{1}} & 0 & 0 \\
0 & 0 & e^{-\beta \epsilon_{1}} & 0 \\
0 & 0 & 0 & e^{\beta \epsilon_{1}}
\end{array}\right)
$$

The internal energy of the system is

$$
\begin{aligned}
& \langle E\rangle=\operatorname{Tr}(\rho H)
\end{aligned}
$$

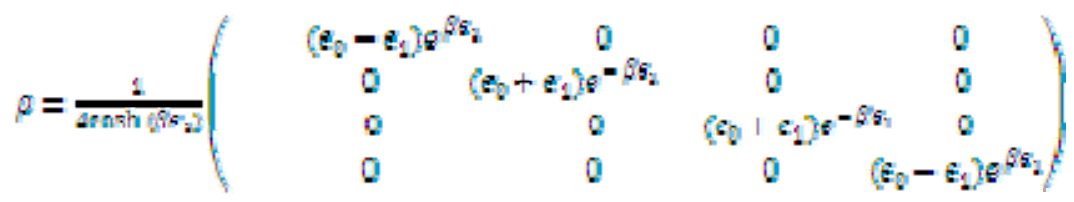

yielding

$$
\langle E\rangle=\epsilon_{0}-\epsilon_{1} \tanh \left(\beta \epsilon_{1}\right)
$$

The energy increases with temperature and approaches a constant value $\epsilon_{0}$ as depicted in figure 6 . The Helmholtz free energy is obtained as

$$
F=\epsilon_{0}-\frac{1}{\beta} \operatorname{In}\left[4 \operatorname{Cosh}\left(\beta \epsilon_{1}\right)\right]
$$

Figure 7 shows a plot of Helmholtz energy with temperature and it shows that $\mathrm{F}$ increases negatively and indefinitely with temperature.

The entropy is calculated using (10) as

$$
S=k_{B} \operatorname{In}\left[4 \operatorname{Cosh}\left(\beta \epsilon_{1}\right)\right]-\frac{\epsilon_{1}}{T} \tan \hbar\left(\beta \epsilon_{1}\right)
$$

The entropy has a minimum value of $0.6952 \mathrm{k}_{\mathrm{B}}$. This shows that the four level state systems is always in a state of disorder. With increasing temperature, the entropy approaches a constant value of $1.381 \mathrm{k}_{\mathrm{B}}$. This also obeys the second law of thermodynamics $(\Delta S \geq 0)$ as shown in figure 8 . 
Finally, we obtained the heat capacity as

$$
C_{V}=k_{z}\left(\frac{e_{1}}{k_{z} T}\right)^{2} \frac{e^{-\beta \varepsilon_{1}}}{\left(1+e^{\left.-2 \beta \varepsilon_{1}\right)^{2}}\right.}=k_{z}\left(\frac{e_{1}}{k_{\sigma} T}\right)^{2} \operatorname{Sech}^{2}\left(\beta e_{1}\right)
$$

The behaviour of equation (44) is shown in figure 9 for $C_{V}$ and it increases between $0 \leq T \leq 1$ and decreases between $1 \leq T \leq \infty$.

Equation (44) can also written as an infinite series as,

$$
C_{v}=k_{\beta}\left(\frac{\epsilon_{1}}{k_{B} T}\right)^{2} \sum_{n=0}^{\infty}\left[(2 n+1) e^{-2(2 n+1) \beta \epsilon_{1}}-2 n e^{-4 n \beta \epsilon_{1}}\right],
$$

and the behavior is depicted in figure 10 .

\section{Conclusion}

We have evaluated the thermodynamics properties of a quantum harmonic oscillator and a four level system via density matrix method. The resulting specific heat capacity approaches the classical (Pettit-Dulong law) result for high temperatures and goes to zero for vanishing temperature. The entropies of both systems also obey the second law of thermodynamics as well as the third law of thermodynamics. The Helmholtz free energies of the systems increase negatively with temperature which is the prediction of thermodynamics. Finally, we obtain the numerical results for the harmonic and four level system and they conform to the known results using the partition function methods.

\section{References}

Davydov A. S. (1991). Quantum Mechanics, Pergamon Press, New York, pp 41-46.

E. Kozliak and F. L. Lambert. (2008). Residual Entropy, the Third law and Latent Heat. Entropy, 10. pp 274-284. doi:10.3390/e10030270, http://dx.doi.org/10.3390/e10030270

Feynman R.P. (1972). Statistical Mechanics, Addison-Wesley.

Hanggi P and Ingold L. (2005). Fundamental Aspects of Quantum Brownian Motion. Chaos. pp. 1-12. doi:10.1063/1.1853631, http://dx.doi.org/10.1063/1.1853631

Hanggi P. and Ingold G. (2006). Quantum Brownian Motion and the Third Law of Thermodynamics. Acta Phys.Pol., B, 37, No. 5, 1537-1550.

Hijar H and de Zarate J. O. (2010). Jarzynski Equality Illustrated by Simple Examples. Euro J. Phys, 31, pp 10971106.

Ingold G, Hanggi P and Talkner P. (2009). Specific Heat Anomalies of Open Quantum Systems. arxiv: quant-ph/0811.3509. doi:10.1103/physRevE.79.061105, http://dx.doi.org/10.1103/physRevE.79.061105

Ingold G, Lambert A and Reynaud S. (2009). Quantum Dissipative Brownian Motion and the Casimir Effect. arxiv: quant-ph/0905.3608. doi:10.1103/PhysRevE.80.041113, http://dx.doi.org/10.1103/PhysRevE.80.041113

Kim S. P, Santana A. E and Khana F. C. (2003). Decoherence of Quantum Damped Oscillators. J. Korean Phys. Soc., 43. No. 4, pp 452-460.

Kittel C. (1988). Introduction to Solid State Physics. Wiley \& Sons, Inc. New York, pp. 100-115.

Landau L. D. and Lifshitz E. M. (1980). Statistical Physics, Pergamon Press, New York, pp 195-198.

Merzbacher E. (1976). Quantum Mechanics; Wiley \& Sons Inc: New York, pp 278-291.

Messiah A. (1970). Quantum Mechanics Vol. 1; Willey \& Sons Inc: New York, pp 331-338.

P. Hanggi and G. L. Ingold. (2008). Acta Phys., Pol. B37 1161.

Sethna J. P. (2006). Entropy, Order Parameters and Complexity, Cladevon Press, Oxford, pp 157-163. 


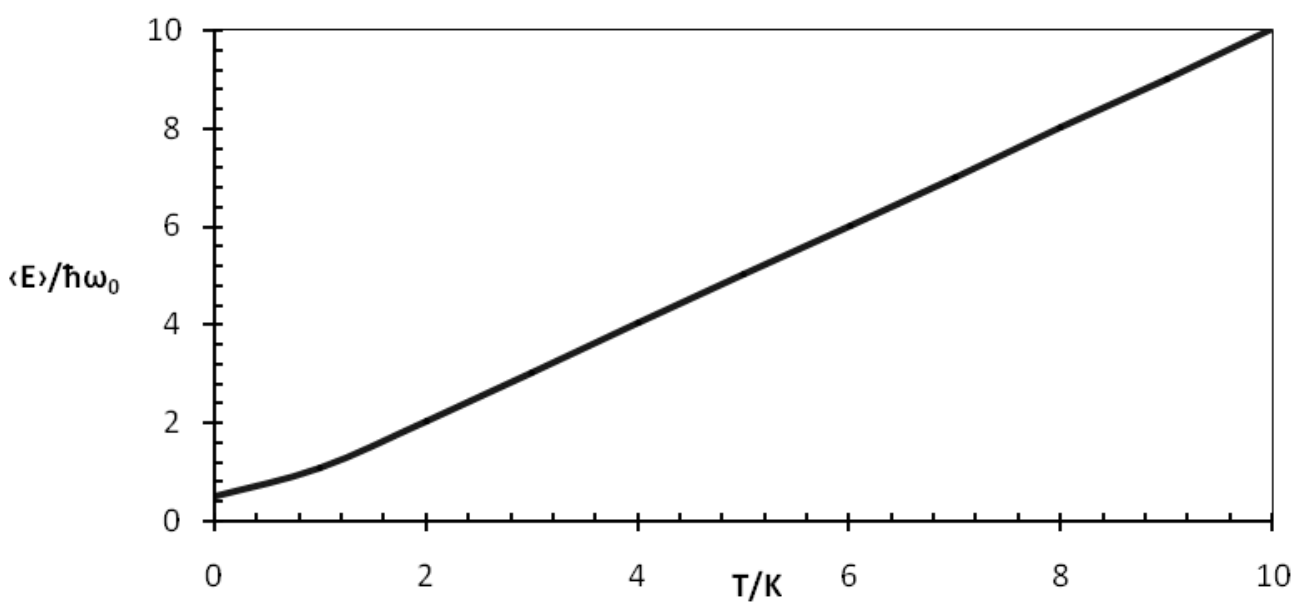

Figure 1. Internal Energy $\langle\mathbf{E}\rangle$ with Temperature $\mathbf{T}$ for the Harmonic Oscillator The relationship gives a linear relationship as predicted by Quantum mechanics.

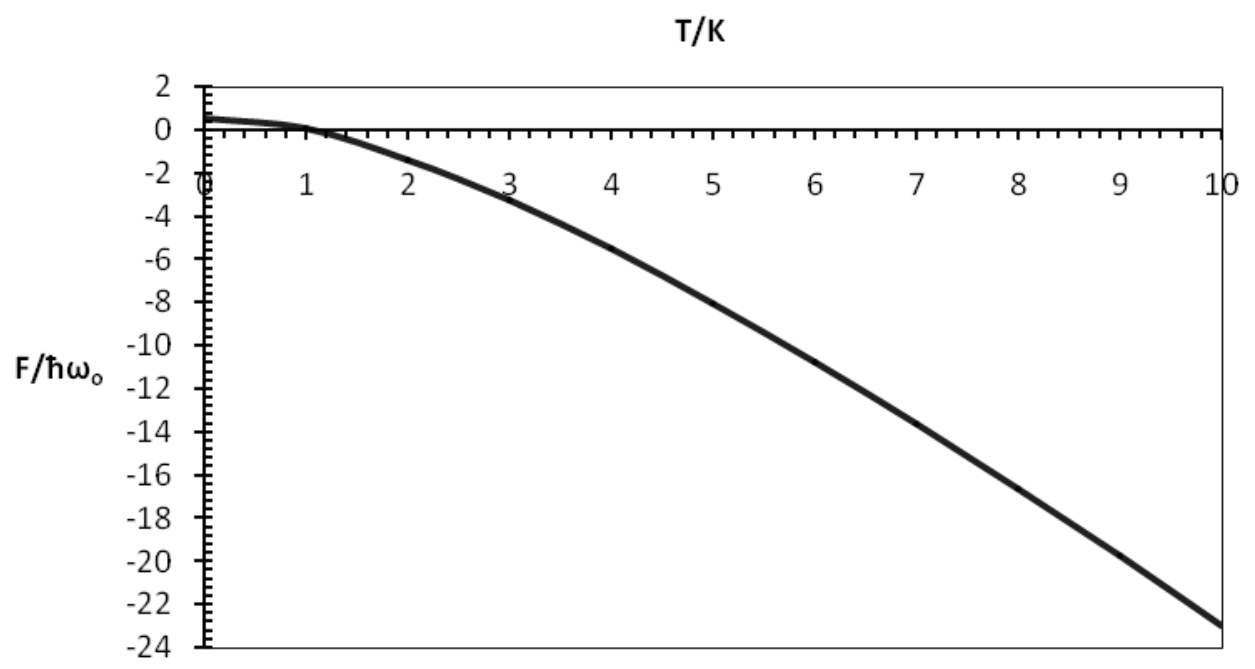

Figure 2. Free Energy $\mathbf{F}$ with Temperature $\mathbf{T}$ for the Harmonic Oscillator As temperature increases the Free energy increases negatively as expected. 


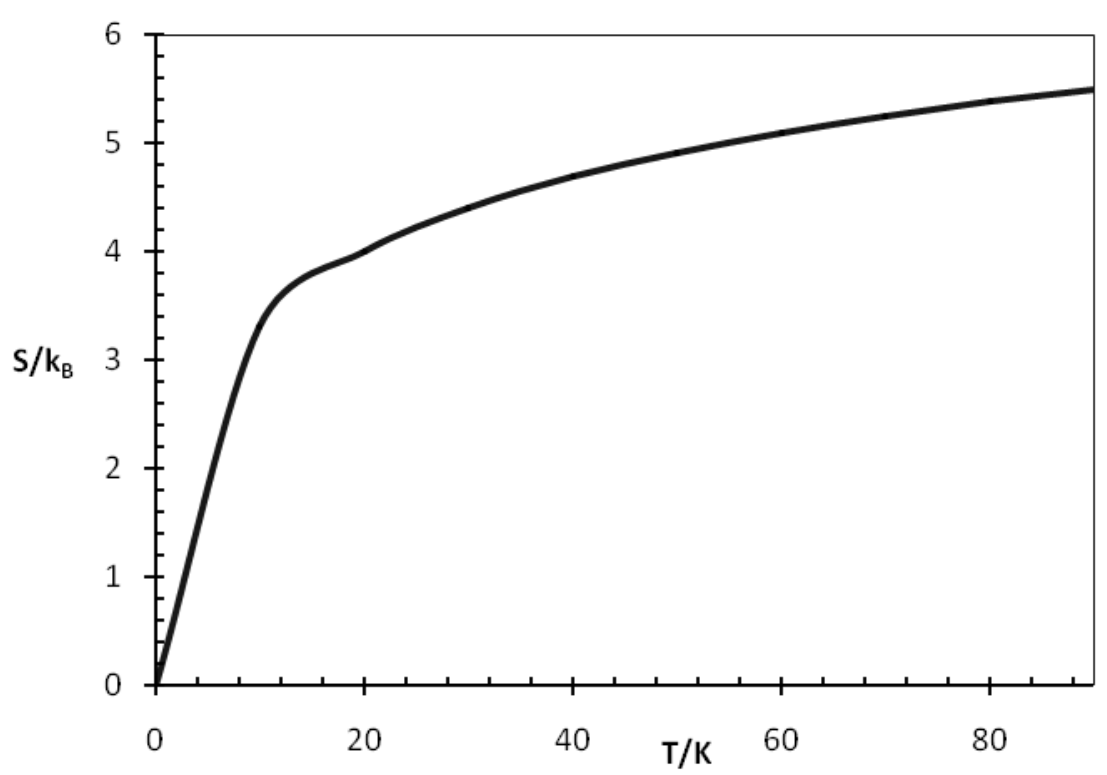

Figure 3. Entropy $\mathbf{S}$ with Temperature $\mathbf{T}$ for the Harmonic Oscillator As $\mathbf{T} \rightarrow 0, \mathbf{S} \rightarrow 0$ obeying the third law of thermodynamics. As $\mathbf{T}$ increases, $\mathbf{S}$ also increases in agreement with the second law of thermodynamics

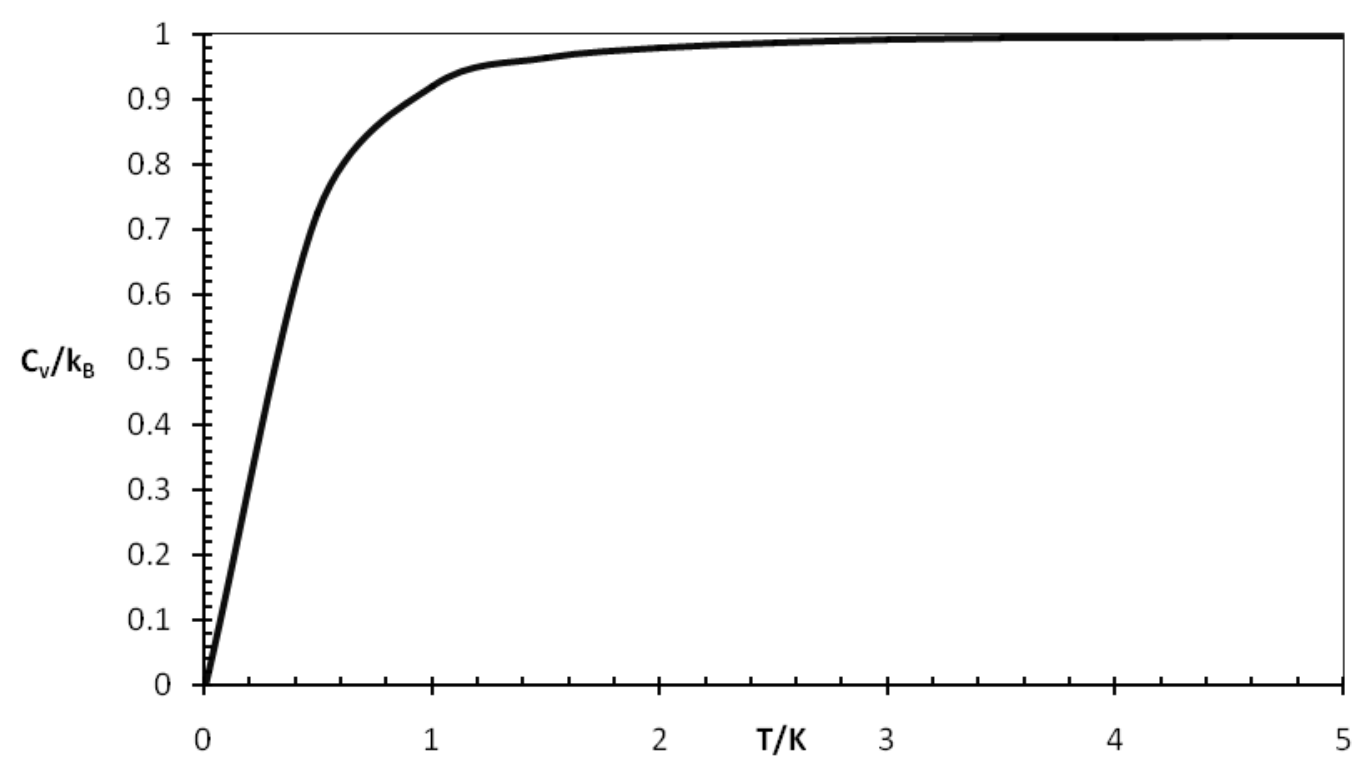

Figure 4. Heat Capacity $\mathbf{C}_{v}$ with Temperature $\mathbf{T}$ for the Harmonic Oscilltor As temperarure increases, the heat cap acity approaches a constant value $\mathbf{k}_{\mathrm{B}}$. It reduces to the classical rule of Dulong and Petit at high temperatures 


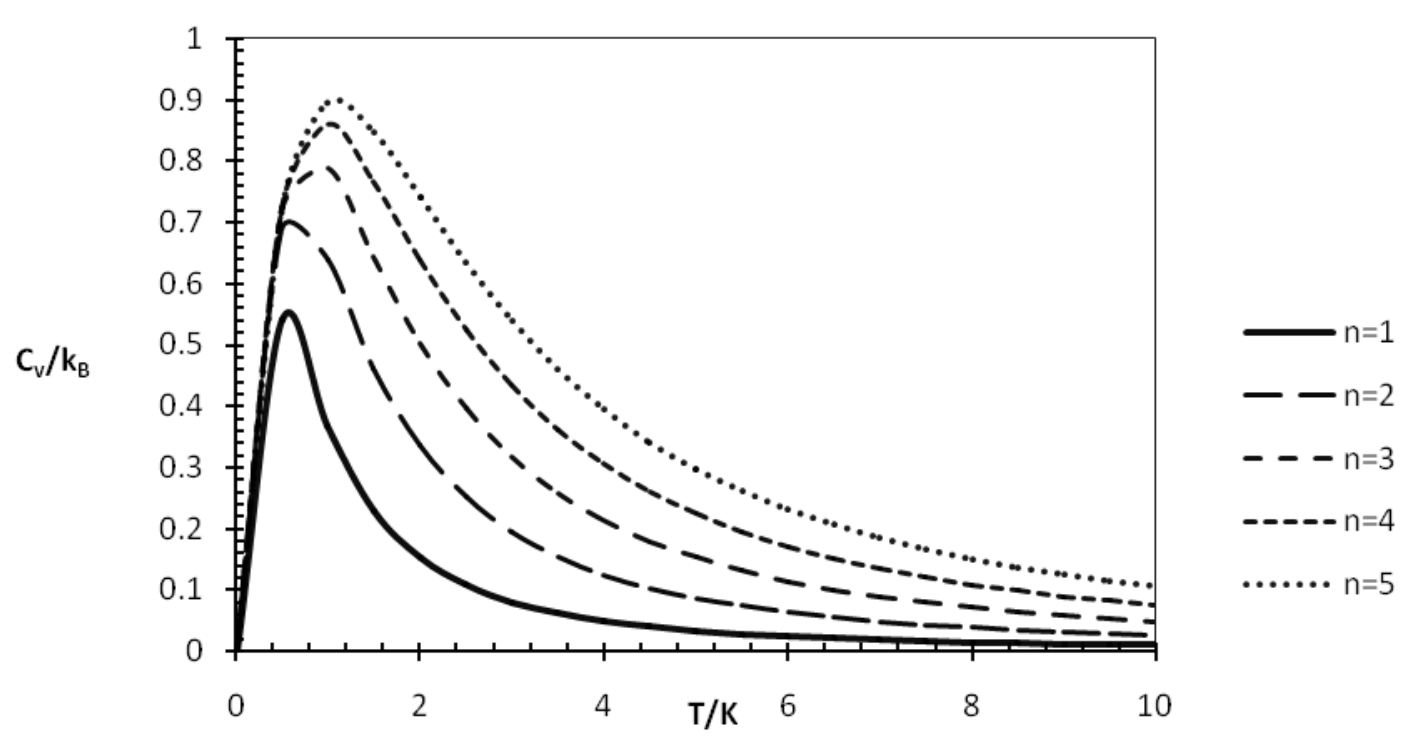

Figure 5. Heat Capacity $\mathbf{C}_{\mathrm{v}}$ with Temperature $\mathbf{T}$ for different values of $\mathbf{n}$ for the Harmonic Oscillator. As the value of $\mathbf{n}$ increases the peak of the $\mathrm{C}_{\mathrm{v}}$ shifts forward, showing that the heat capacity increase as $n$ increases

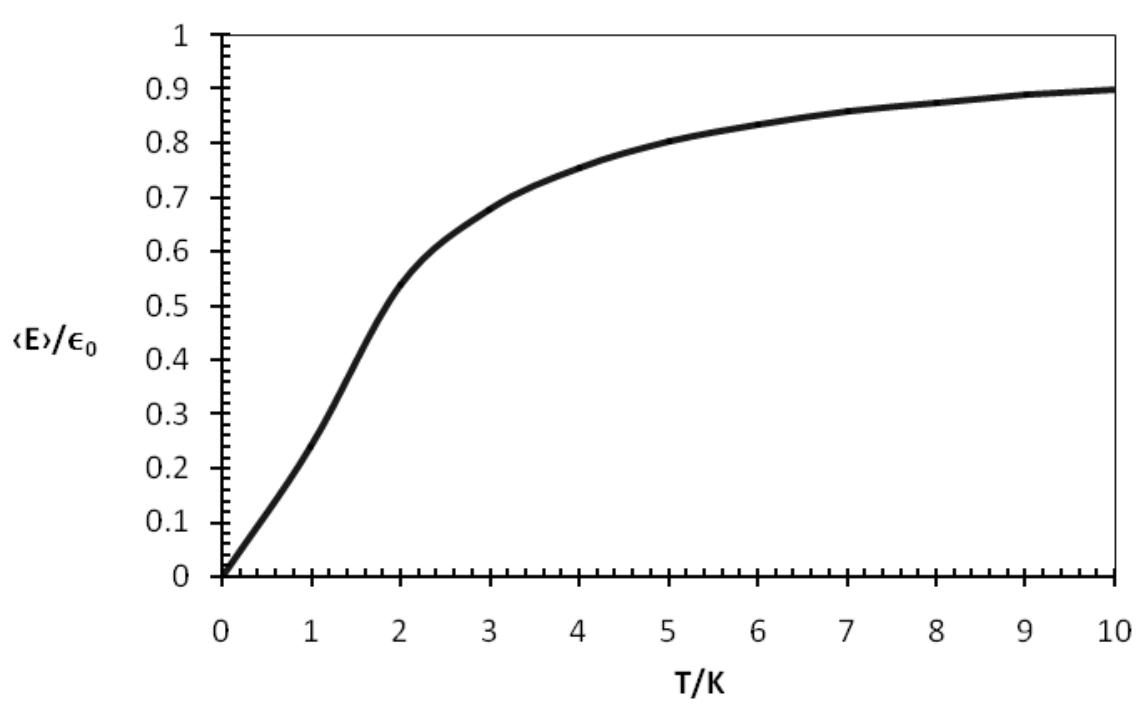

Figure 6. Internal Energy 〈E〉 with Temperature $\mathbf{T}$ for the Four Level System. The internal energy increases with temperature. It has maximum value of $\epsilon_{\mathbf{0}}$ 


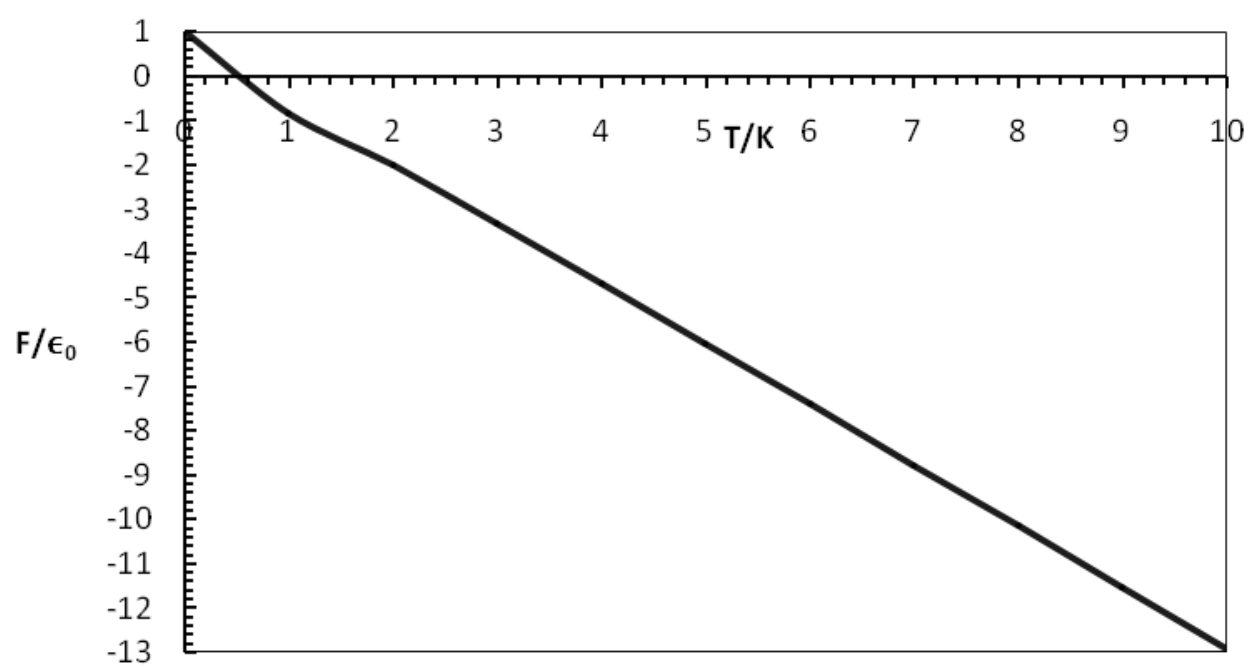

Figure 7. Free Energy $\mathbf{F}$ with Temperature $\mathbf{T}$ for the Four Level System.

Just like the free energy of the Harmonic Oscillator and in accordance to the predictions of Thermodynamics, the free energy of the four level system increases negatively with temperature

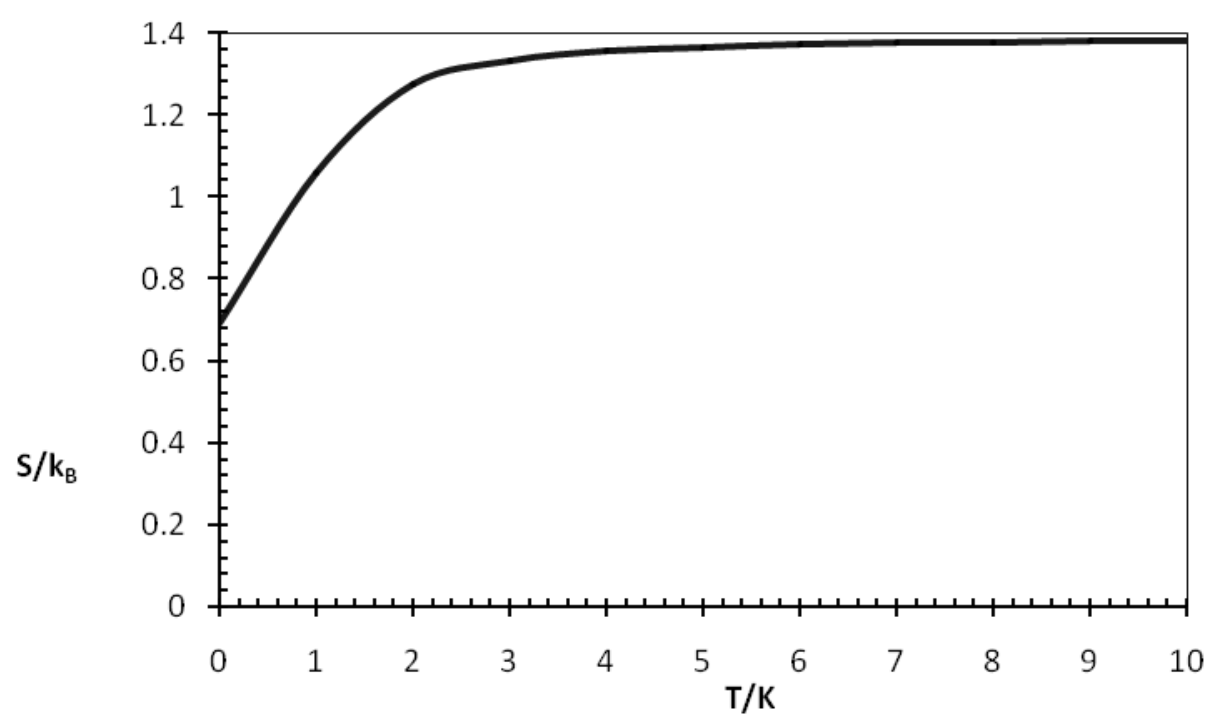

Figure 8. Entropy $\mathbf{S}$ with Temperature $\mathbf{T}$ for the Four Level System. The entropy initially increases from a minimum value at $\mathbf{T}=0$ and then later approaches a constant value. There is a minimum entropy greater than zero even when the temperature of the system is zero. The system still obeys the second law of thermodynamics since $\Delta S \geq 0$ as shown 


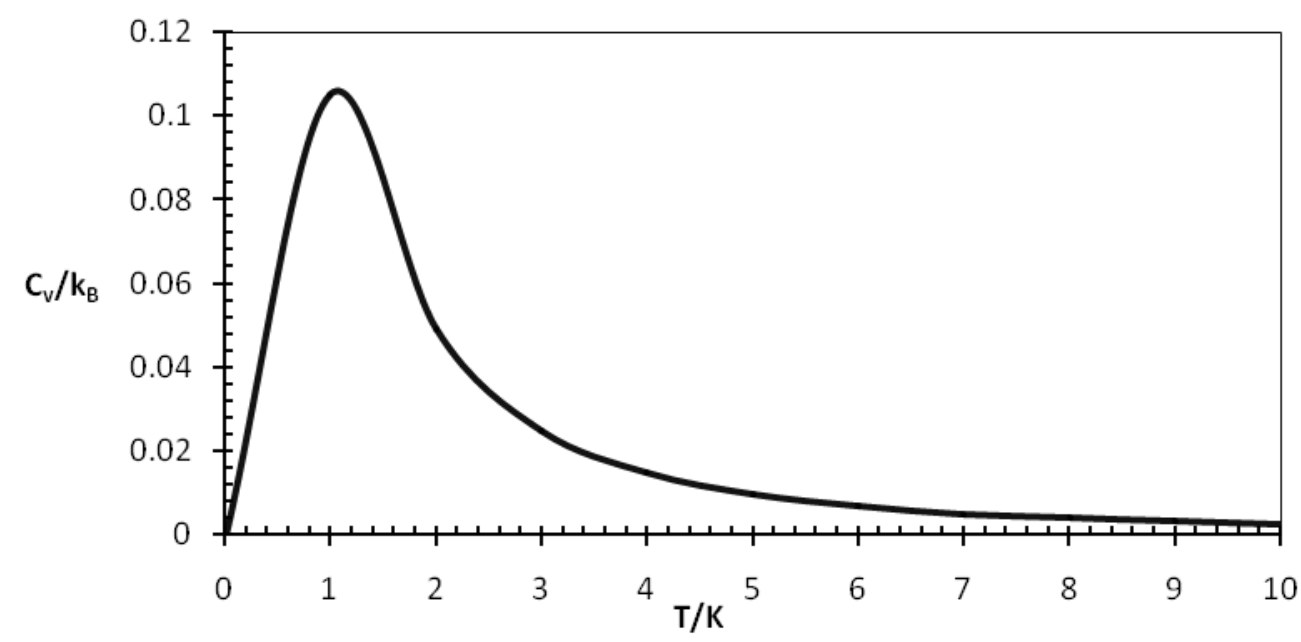

Figure 9. Heat Capacity $\mathbf{C}_{\mathbf{v}}$ with Temperature $\mathbf{T}$ for the Four Level System

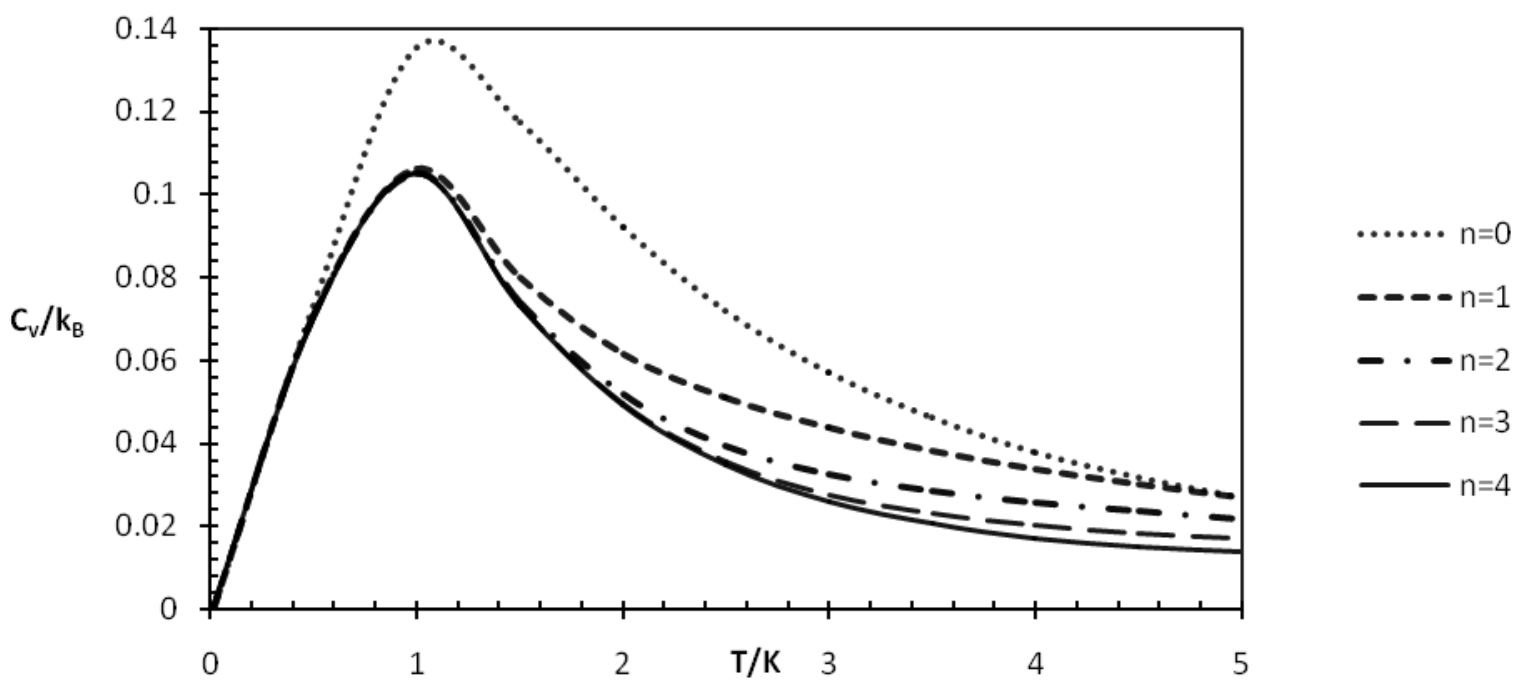

Figure 10. Heat Capacity $\mathbf{C}_{\mathrm{v}}$ with Temperature $\mathbf{T}$ for the Four Level System for different values of $\mathbf{n}$. The heat capacity increases with $\mathbf{n}$ which means that higher states have higher heat capacities 